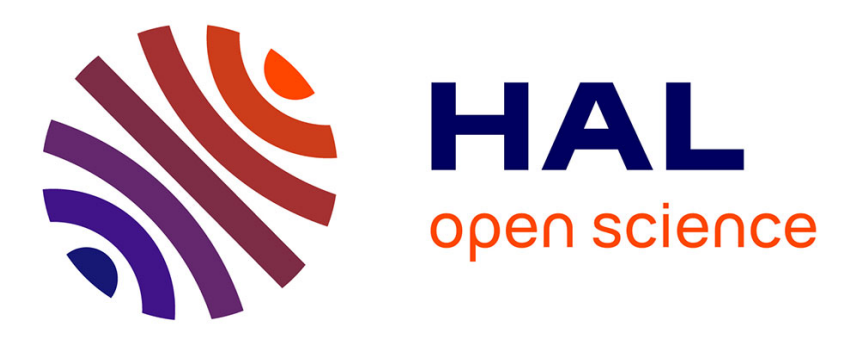

\title{
Properties of the $67 \mathrm{P} /$ Churyumov-Gerasimenko interior revealed by CONSERT radar
}

\author{
Wlodek Kofman, Alain Herique, Yves Barbin, Jean-Pierre Barriot, Valérie \\ Ciarletti, Stephen Clifford, Peter Edenhofer, Charles Elachi, Christelle \\ Eyraud, Jean-Pierre Goutail, et al.
}

\section{To cite this version:}

Wlodek Kofman, Alain Herique, Yves Barbin, Jean-Pierre Barriot, Valérie Ciarletti, et al.. Properties of the 67P/Churyumov-Gerasimenko interior revealed by CONSERT radar. Science, 2015, 349 (6247), pp.aab0639. 10.1126/science.aab0639 . insu-01182590

\section{HAL Id: insu-01182590 \\ https://hal-insu.archives-ouvertes.fr/insu-01182590}

Submitted on 18 Nov 2020

HAL is a multi-disciplinary open access archive for the deposit and dissemination of scientific research documents, whether they are published or not. The documents may come from teaching and research institutions in France or abroad, or from public or private research centers.
L'archive ouverte pluridisciplinaire HAL, est destinée au dépôt et à la diffusion de documents scientifiques de niveau recherche, publiés ou non, émanant des établissements d'enseignement et de recherche français ou étrangers, des laboratoires publics ou privés. 


\title{
Properties of the
}

\section{P/Churyumov-Gerasimenko interior revealed by CONSERT radar}

\author{
Wlodek Kofman, ${ }^{1}$ Alain Herique, ${ }^{1}$ Yves Barbin, ${ }^{2}$ Jean-Pierre Barriot, ${ }^{3}$ Valérie Ciarletti, \\ Stephen Clifford, ${ }^{5}$ Peter Edenhofer, ${ }^{6}$ Charles Elachi, ${ }^{7}$ Christelle Eyraud, ${ }^{15}$ \\ Jean-Pierre Goutail, ${ }^{4}$ Essam Heggy, ${ }^{7,17}$ Laurent Jorda, ${ }^{12}$ Jérémie Lasue, ${ }^{14}$ \\ Anny-Chantal Levasseur-Regourd, ${ }^{13}$ Erling Nielsen, ${ }^{8}$ Pierre Pasquero, ${ }^{1}$ \\ Frank Preusker, ${ }^{16}$ Pascal Puget, ${ }^{1}$ Dirk Plettemeier, ${ }^{9}$ Yves Rogez, ${ }^{1}$ Holger Sierks, ${ }^{8}$ \\ Christoph Statz, ${ }^{9}$ Hakan Svedhem, ${ }^{10}$ Iwan Williams, ${ }^{11}$ Sonia Zine, ${ }^{1}$ Jakob Van Zyl ${ }^{7}$
}

The Philae lander provides a unique opportunity to investigate the internal structure of a comet nucleus, providing information about its formation and evolution in the early solar system. We present Comet Nucleus Sounding Experiment by Radiowave Transmission (CONSERT) measurements of the interior of Comet 67P/Churyumov-Gerasimenko. From the propagation time and form of the signals, the upper part of the "head" of 67P is fairly homogeneous on a spatial scale of tens of meters. CONSERT also reduced the size of the uncertainty of Philae's final landing site down to approximately 21 by 34 square meters. The average permittivity is about 1.27 , suggesting that this region has a volumetric dust/ice ratio of 0.4 to 2.6 and a porosity of 75 to $85 \%$. The dust component may be comparable to that of carbonaceous chondrites.

ong-wavelength radars are currently extensively used to study the subsurface of planetary bodies down to a few kilometers in depth, examples being the Mars Advanced Radar for Subsurface and Ionospheric Sounding on the Mars Express European Space Agency (ESA) mission (1,2), Shallow Radar on the Mars Reconnaissance Orbiter NASA mission (3), and Lunar Radar Sounder on the Kaguya Japan Aerospace Exploration Agency mission (4). Because comet nuclei are on the order of a few kilometers in size and made of highly porous material, we modified the above technique to study the nucleus of a comet. The Comet Nucleus Sounding Experiment by Radiowave Transmission (CONSERT) was first proposed in 1994 and selected by ESA in 1996 as one of the experiments on the Rosetta mission. It was initially intended to explore comet 46P/Wirtanen, but, due to delay in the launch date, the target had to be changed to comet $67 \mathrm{P} /$ Churyumov-Gerasimenko (67P), which has a larger nucleus.

CONSERT (5), a bistatic radar instrument, propagates long-wavelength electromagnetic signals between the orbiting Rosetta spacecraft and the lander, Philae. In general, part of the signal path travels through the nucleus [see the supplementary materials (SM)]. The measured quantities are the signal travel time and the amplitude of the received signals. The travel time depends on the real part of the permittivity (dielectric constant), whereas the imaginary part of the permittivity (linked to the electrical conductivity) has an effect on the signal's amplitude. Thus, the CONSERT measurements give direct information about the permittivity of the comet nucleus and its spatial structure. The permittivity is a function of several properties of the nucleus: porosity, composition of the material, temperature, internal structure, and/or scale of potential heterogeneities. Theoretical models of the internal structure of the comet have been produced encompassing a range of values for the above parameters to obtain a match to the observed time delay and amplitude of the signals, thus allowing conclusions to be drawn regarding the interior of the comet nucleus.

\section{Measurements during the First Science Sequence}

Philae separated from the Rosetta orbiter at 08:35 UTC on 12 November 2014. CONSERT operated throughout the descent of Philae until 14:51 UTC, 40 min before the scheduled touchdown on the surface of comet 67P. Unfortunately, Philae bounced a couple of times before finally coming to rest in an unknown location and an unknown orientation at 17:31 UTC. CONSERT restarted operating at 18:56 UTC and continued until 04:06 UTC on 13 November 2014. The whole series of observations carried out by Philae after landing is named the First Science Sequence (FSS).

The observations to be made by CONSERT were predetermined long before the landing. It was intended that Philae and Rosetta be initially visible to each other so that, for calibration purposes, the signal would travel only through vacuum. As Rosetta moved about its orbit and the comet nucleus rotated, the geometry would evolve so that an occultation would occur (that is, the nucleus would be between Philae and Rosetta), so that the radio waves would travel through the nucleus. Because Philae was not at the intended landing point, normal communication between Philae and Rosetta was impossible at that time, implying that there was no direct visibility between the communication antennas. Then, Philae and Rosetta were already in occultation when the measurements started. Hence, there was no direct visibility for CONSERT either, making calibration difficult.

Because Rosetta moves along its orbit, while at the same time the nucleus rotates, the relative positions of Rosetta and Philae were continually changing during the FSS (Fig. 1). Both the path length and its trajectory were changing. A strong signal was detected for about $30 \mathrm{~min}$ (18:56 to 19:22 UTC) at the beginning of the FSS and for about $80 \mathrm{~min}$ at the end of the FSS $(02: 47$ to 04:06 UTC) (Fig. 2). During these two periods, the signals at both Philae and Rosetta were strong and CONSERT worked as intended (SM). The results presented in the paper are mainly based on the data acquired during these two periods.

Outside these two periods, the observed signalto-noise ratio (SNR) was much lower. This can be explained by some of following factors: (i) the FSS orbit was not well adapted to the CONSERT bistatic measurements; (ii) the lander antennas were not well positioned with respect to the local environment at the surface (resulting in a gain loss and a polarization mismatch between the lander and orbiter); (iii) the noise level at the receiver on Philae during the FSS measurements was much larger (about $12 \mathrm{~dB}$ ) than

Université Grenoble Alpes, IPAG, F-38000 Grenoble, France (2) Centre National de la Recherche Scientifique (CNRS), Institut de Planétologie et d'Astrophysique de Grenoble (IPAG), F-38000 Grenoble, France. ${ }^{2}$ MIO, UM 110, CNRSInstitut National des Sciences de l'Univers (INSU), Université de Toulon, Aix-Marseille Université, IRD 83957 La Garde, France. ${ }^{3}$ Geodesy Observatory of Tahiti BP6570, 98702 Faa'a, Tahiti. ${ }^{4}$ Université de Versailles Saint-Quentin-enYvelines (UVSQ) (UPSay); Université Pierre et Marie Curie (UPMC) (Sorbonne Univ.); CNRS/INSU; Laboratoire Atmosphéres, Milieux, Observations Spatiales (LATMOS)Institut Pierre-Simon Laplace (IPSL), 11 Boulevard d'Alembert, 78280 Guyancourt, France. ${ }^{5}$ Lunar and Planetary Institute, 3600 Bay Area Boulevard, Houston, TX 77058, USA. ${ }^{6}$ Ruhr-University of Bochum, Faculty of Electrical Engineering and Information Technology, 44780 Bochum, Germany. 7 Jet Propulsion Laboratory, 4800 0ak Grove Drive, MS 300-243E, Pasadena, CA 91109, USA. ${ }^{8}$ MaxPlanck-Institüt fur Sonnensystemforschung (MPS), Justusvon-Liebig-Weg 3, 37077 Göttingen, Germany. ${ }^{9}$ Technische Universitaet Dresden Helmholtzstraße 10 D-01069 Dresden, Germany. ${ }^{10}$ European Space Agency (ESA)/European Space Research and Technology Centre (ESTEC) Noordwijk, Netherlands. ${ }^{11}$ Queen Mary University of London, Mile End Road, London E1 4NS, UK. ${ }^{12}$ Laboratoire d'Astrophysique de Marseille Pôle de l'Étoile Site de Château-Gombert 38, Rue Frédéric Joliot-Curie 13388 Marseille, France. ${ }^{13}$ UPMC (Sorbonne Univ.); UVSQ (UPSay); CNRS/INSU; LATMOSIPSL, BC 102, 4 place Jussieu, 75005 Paris, France. ${ }^{14}$ Université de Toulouse; UPS-OMP. IRAP; (2) CNRS; IRAP; 9 Avenue Colonel Roche, BP 44 346, F-31028 Toulouse Cedex 4, Toulouse, France. ${ }^{15}$ Aix-Marseille Université, CNRS Centrale Marseille, Institut Fresnel UMR 7249, 13013 Marseille, France. ${ }^{16}$ German Aerospace Center (DLR) Rutherfordstraße 212489 Berlin, Germany. ${ }^{17}$ University of Southern California, Ming Hsieh Department of Electrical Engineering, Viterbi School of Engineering, Los Angeles, CA 90089, USA

*Corresponding author. E-mail: wlodek.kofman@obs.ujf-grenoble.fr 
the one measured during the cruise phase; and (iv) the absorption inside the comet, linked to the length of propagation path and electrical properties of the nucleus, could also contribute to decrease the signal power. Fortunately enough, since the noise level at the receiver on Rosetta was much lower than the one experienced at the receiver on Philae, signals could still be clearly measured on Rosetta during some additional

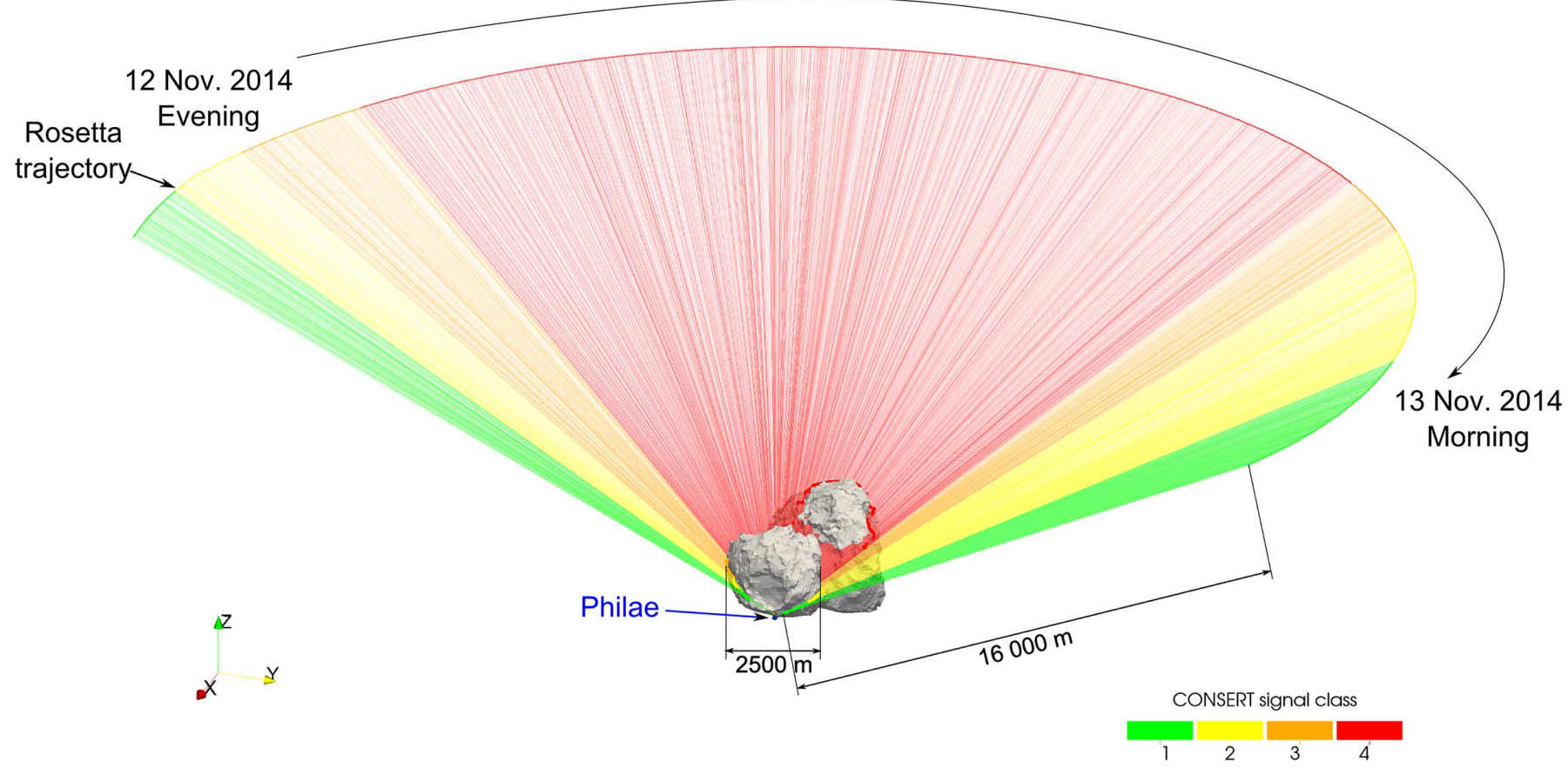

Fig. 1. Propagation of signals from Philae on the nucleus to Rosetta on its orbit. The orbit of Rosetta and the location of Philae inside the strip determined by CONSERT are shown. The rotation of the nucleus dominates the relative motion of Rosetta versus Philae. Different colors for the propagation lines correspond to different qualities of CONSERT data. The class of the signal is color-coded in (1) green for strong SNR and good synchronization, (2) yellow for acceptable SNR without synchronization, (3) orange for low SNR, and (4) red for absence of signal. In the figure, we indicated on the comet nucleus model the lines (ground track) where the signals from Philae that go to Rosetta cross the surface of the comet. The width of the antenna beam is about $78^{\circ}$, and its footprint covers the whole comet. Figures S1 and $\mathrm{S} 2$ show closer views of the ground tracks. The green lines show the locations of Rosetta during the period when the measurements we discuss in this paper were obtained.
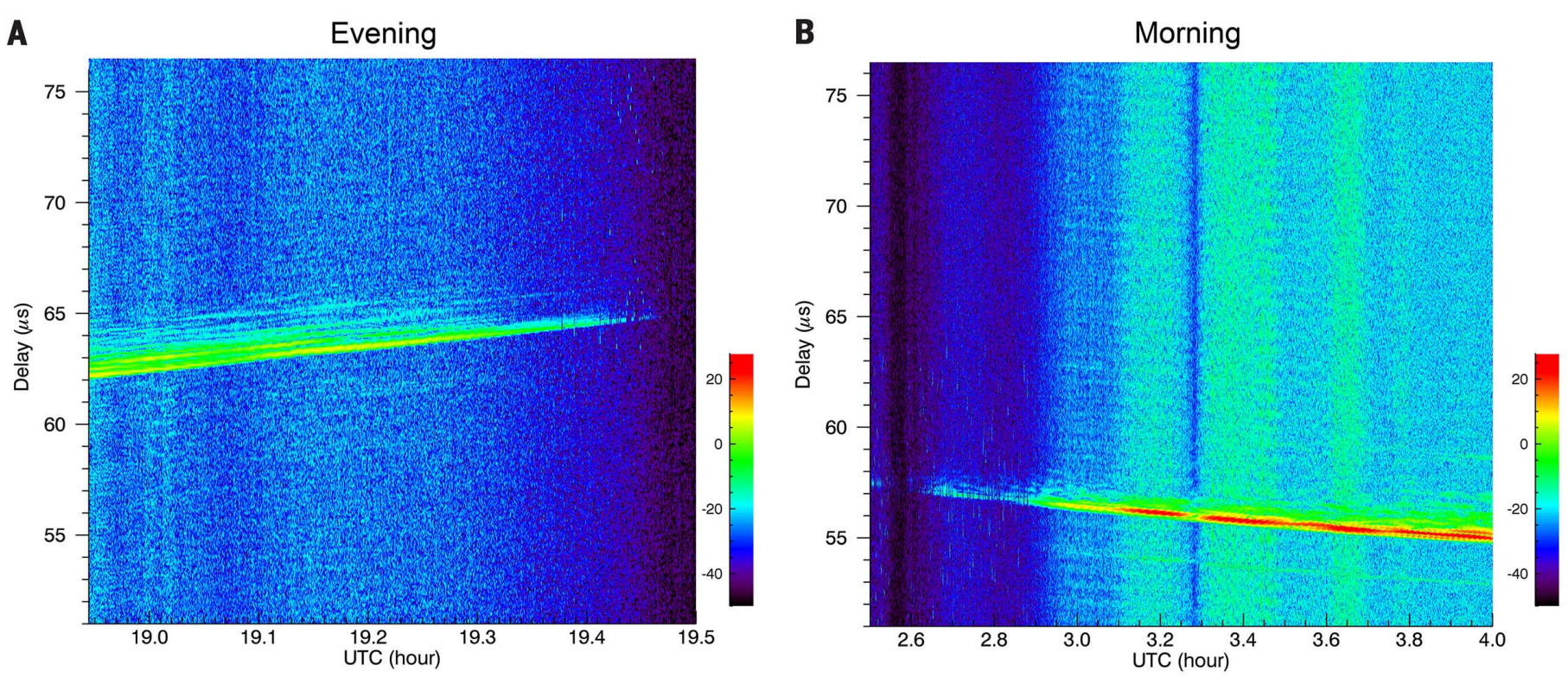

Fig. 2. The signal power in $\mathbf{d B}$ or in logarithmic scale on 12 to 13 November 2014. Times on (A) 12 November (evening) and (B) 13 November (morning) are in universal time coordinated (UTC); for the same diagram expressed in a linear power scale, see the SM. The figure shows the signal received as a function of propagation time between Philae and Rosetta in $\mu \mathrm{s}$ (vertical axis) and time of measurements UTC (every $2.5 \mathrm{~s}$ ) (horizontal axis). The color code indicates the power of the signal (red being the strongest). 
periods despite a partial loss of synchronization, so that useful results could be obtained. Another measurement period corresponds to a situation in which the statistics of the signal at Rosetta show the presence of a signal that propagated through the comet but where a clear detection is not possible because the signal is less powerful than the noise.

CONSERT is able to measure the distance between Philae and Rosetta when they are visible to each other simply by multiplying the signal travel time by the speed of the radio wave in vacuum. Because Philae's location was unknown, CONSERT carried out three additional measurements while Rosetta and Philae were visible to each other, in an attempt to locate the position of Philae on the surface by triangulation. The triangulation was made during three additional measurements of about 15 min each, on 13 November at 22:00 UTC and on 14 November at 10:30 and 23:45 UTC. The location of Philae was pinned down to a strip measuring approximately 150 by $15 \mathrm{~m}^{2}$, with accuracies on the order of 10 to $20 \mathrm{~m}(6)$

Signals that were received by CONSERT on Rosetta during the two nominal periods of measurements propagated through parts of the nucleus and are as narrow as the calibration signal (Fig. 3). Thus, we conclude that there is not, at least down to the level of $20 \mathrm{~dB}$ below the maximum of the peak, any signature of volume or surface scattering effects in the signal form. If scattering were present, a long tail of decay in the signal should be visible (7). The absence of this scattering indicates that the medium explored by the waves is rather homogeneous and/or that the dielectric contrast (difference) between potential inclusions inside the nucleus is low, at least at the scale of a few wavelengths of CONSERT. This conclusion is important for our approach to the data interpretation.

This result does not, however, exclude a slow variability in the dielectric properties or the existence of blocks much larger than the wavelength inside the nucleus. Two or three well-defined propagation paths could indeed be potentially due to the presence of a large structure inside the nucleus or to surface features on a large scale (Fig. 3 and fig. S3).

We processed and analyzed the data to determine the propagation time between Philae and Rosetta (Fig. 4). Had the operations been normal, a propagation time in free space would have been calculated, and the difference with the measured time combined with the knowledge of the nucleus shape model would have led to the determination of average dielectric properties of the materials. In reality, because the position of Philae was only known to be within a strip measuring approximately 150 by $15 \mathrm{~m}^{2}$, the exact propagation time in free space cannot be calculated, and this straightforward approach to data analysis could not be used.

Instead, we had to assume a series of potential lander locations within the strip (Fig. 5), larger than mentioned above, in order to take into account the possible inaccuracies, and a range of realistic permittivity values for the nucleus. For every combination, we calculate the propagation time and compare it with the values obtained by CONSERT so that the best matches can be identified.

To analyze the dielectric properties of $67 \mathrm{P}$, a considerable amount of data, both from groundbased observations and other instruments on Rosetta, is already available. This allows restrictions to be placed on the values of the parameters that had to be considered. The measured low average density of the nucleus indicates that the porosity is very high (70 to $80 \%$ ) (8). Nuclei are composed of ices, mainly $\mathrm{H}_{2} \mathrm{O}, \mathrm{CO}$, and $\mathrm{CO}_{2}(9)$, and of refractory dust particles, mostly silicate material and nonvolatile macromolecular material $(8,10)$, already detected by Rosetta $(11,12)$. From these facts and values for the real part of the permittivity of ices and the expected dust-to-ices ratio, using a mixing law (13), we deduce that the permittivity of the cometary interior should be low, much less than 2 , with a low imaginary part. The Wentzel, Kramers, and Brillouin (WKB) $(14,15)$ approximation is valid for a wave propagation model inside the nucleus when the spatial variations of the permittivity are smooth. This requires a scale length $L$, to be much larger than the wavelength (16), and a relative variability $\Delta \varepsilon / \varepsilon$ less than $10 \%$ where $\varepsilon$ is the permittivity. The scale length is defined by $L=\varepsilon /|\nabla \varepsilon|$. Using the Born (14) approximation to estimate the propagation of waves, it is reasonable to assume that the permittivity, with a potential addition of a small perturbation, is constant inside the part of the nucleus explored by CONSERT. This implies that the deviation from a straight line of the propagation path inside the comet is low (16). For a zero-order data analysis, we assumed that the path is a straight line. However, account has to be taken of refraction at the surface due to the difference between the dielectric properties of the nucleus and free space. We ran simulations (SM) of the signal propagation between Philae and Rosetta, taking into account the three-dimensional (3D) shape model provided by the Optical, Spectroscopic, and Infrared Remote Imaging System (OSIRIS) team (shape 4S v0.2 model) (8).

For each of the Rosetta positions on its orbit and for each set of hypothetical Philae position and range of parameters, these 3D simulations produced a set of predicted signals that would be observed, together with their propagation time and propagation paths both inside and outside the comet. These simulations were carried out for 243 hypothetical landing sites along the strip defined for Philae potential position, assuming a constant average permittivity inside the comet. Values of the permittivity ranging from 1.025 to 1.45 have been considered for the simulations due to the low 67P density (8) (SM).

For each simulation, we compare the predicted fastest ray-propagation times with the measured one. The accuracy in time in any one measurement is better than $0.1 \mu \mathrm{s}$, so any hypothetical configuration for which the difference between measured and calculated time is larger than $\pm 0.2 \mu \mathrm{s}$ is rejected. This corresponds to a 60 -m difference in the optical propagation path between Rosetta and Philae. Comparisons between the simulated and measured data have been made separately for each of the two nominal periods of the FSS corresponding to the evening and the morning (figs. S1 and S2). This was done so that any difference in the results could be investigated.

For each Rosetta position on the orbit and for each potential Philae location, the permittivity value that leads to the best match with the experimental data [i.e., minimizes the root mean square (RMS) difference between experimental and simulated delays] can be determined (SM) (figs. S4 and S5). The data taken in the evening show a higher sensitivity to the permittivity, which is consistent with a larger length of the propagation path inside the comet. Eventually, to constrain as much as possible both the location of Philae and the mean permittivity value, we assumed that the mean permittivity value should be the same inside the volume investigated during the morning and evening period. In the evening sector, the length of the propagation path inside the comet is between 560 and $760 \mathrm{~m}$ for all measurements, and in the morning, the variability of length is bigger, 190 to $710 \mathrm{~m}$, most measurements being for smaller lengths. The inferred permittivity range is $1.27 \pm 0.05$ for the evening measurements and $1.27 \pm 0.1$ for the morning ones. The permittivy is normalized with respect to the value for free space. The error was determined assuming that the root mean square deviation on the delays, varies around the minimum by two times the average accuracy of time measurements $(20 \mathrm{~ns})$. The results also lead to the area of the possible locations for the lander of $\sim 21$ by $34 \mathrm{~m}^{2}$ (Fig. 5 ).

\section{Interpretation of the permittivity measurements}

To deduce the bulk nucleus permittivity from the CONSERT results, the effective permittivity of various ices and dust mixtures with different porosities were calculated using mixing formulas and making assumptions regarding the dust and ice ratio (tables S3 to S5). This gives a range of ice/dust volume fraction compatible with the CONSERT values deduced for the mean permittivity.

The permittivity of hexagonal water ice at low temperature is 3.1 at $90 \mathrm{MHz}(17,18)$. The permittivity of amorphous ice, the presence of which in the comets was postulated $(9,19)$, is 3.1 to 3.4 for frequencies of $100 \mathrm{kHz}(20)$. As water ice, whether in its crystalline or amorphous phases, it is nondispersive for frequencies from $\mathrm{kHz}$ to GHz; similar values can be expected at $90 \mathrm{MHz}$. In this analysis, we use a value of 3.1 to estimate the effect of the lower limit of amorphous ice. In the literature, the permittivity of $\mathrm{CO}_{2}$ is given as 2.1 at $200 \mathrm{~K}$ in the 1-MHz frequency range (21). We are unaware of any published measurements for $\mathrm{CO}$. In this analysis, we considered two boundary conditions on the permittivity for the ice fraction component. The first is a permittivity of 3.1 corresponding to the case of pure water ice [upper limit (SM)], and the second is a permittivity of 
2.83 corresponding to a mixture of $75 \% \mathrm{H}_{2} \mathrm{O}$ amorphous ice (or crystalline ice) and $25 \% \mathrm{CO}_{2}$ ice volume ratio corresponding to $\sim 80 \% / 20 \%$ molecular abundances in comets and interstellar material (22). These limits are estimated using the Hashin-Shtrikman bounds (13).

Fig. 3. CONSERT signals as a function of time delay. Signals that have propagated through the nucleus, as measured at the output of the matched filter, are presented for different measurement times and compared to the calibration signal during the cruise phase.

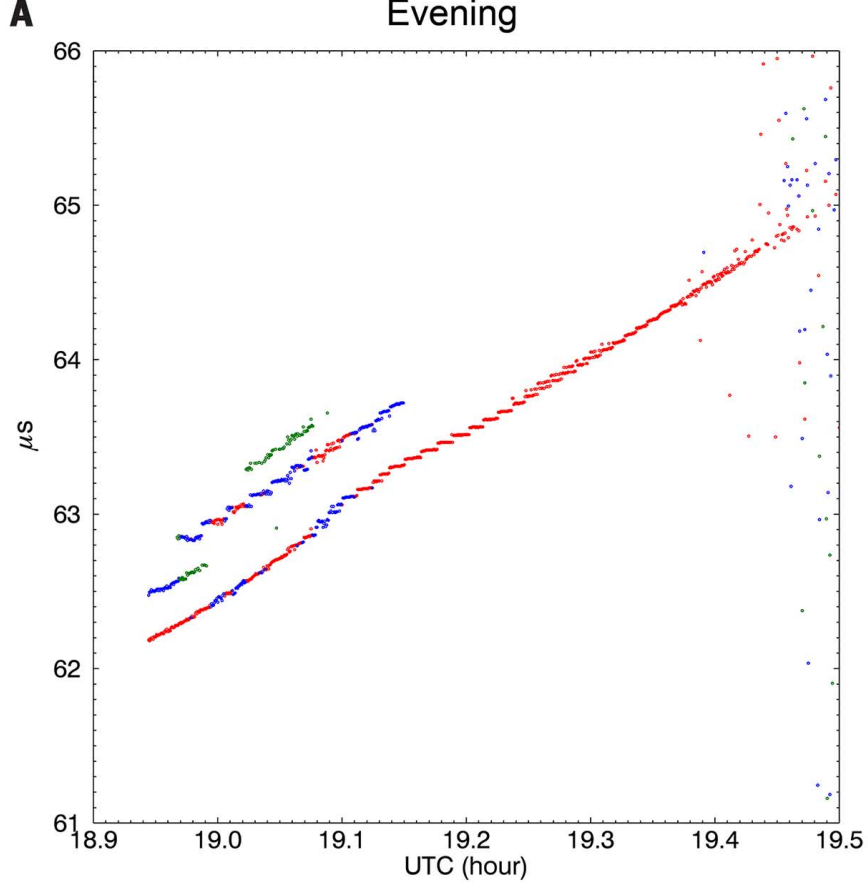

The non-icy fraction of the material plays an important role in modifying the dielectric properties. Ground-based observations suggest that most cometary dust is an unequilibrated, heterogeneous mixture of crystalline and glassy silicate minerals, organic refractory material, and other constituents such as iron sulfides and FeNi metal (23)

The mean elemental composition of comet Wild 2 samples (collected by Stardust) suggests a CI (i.e., similar to carbonaceous chondrite meteorites such as Ivuna meteorite)-like composition
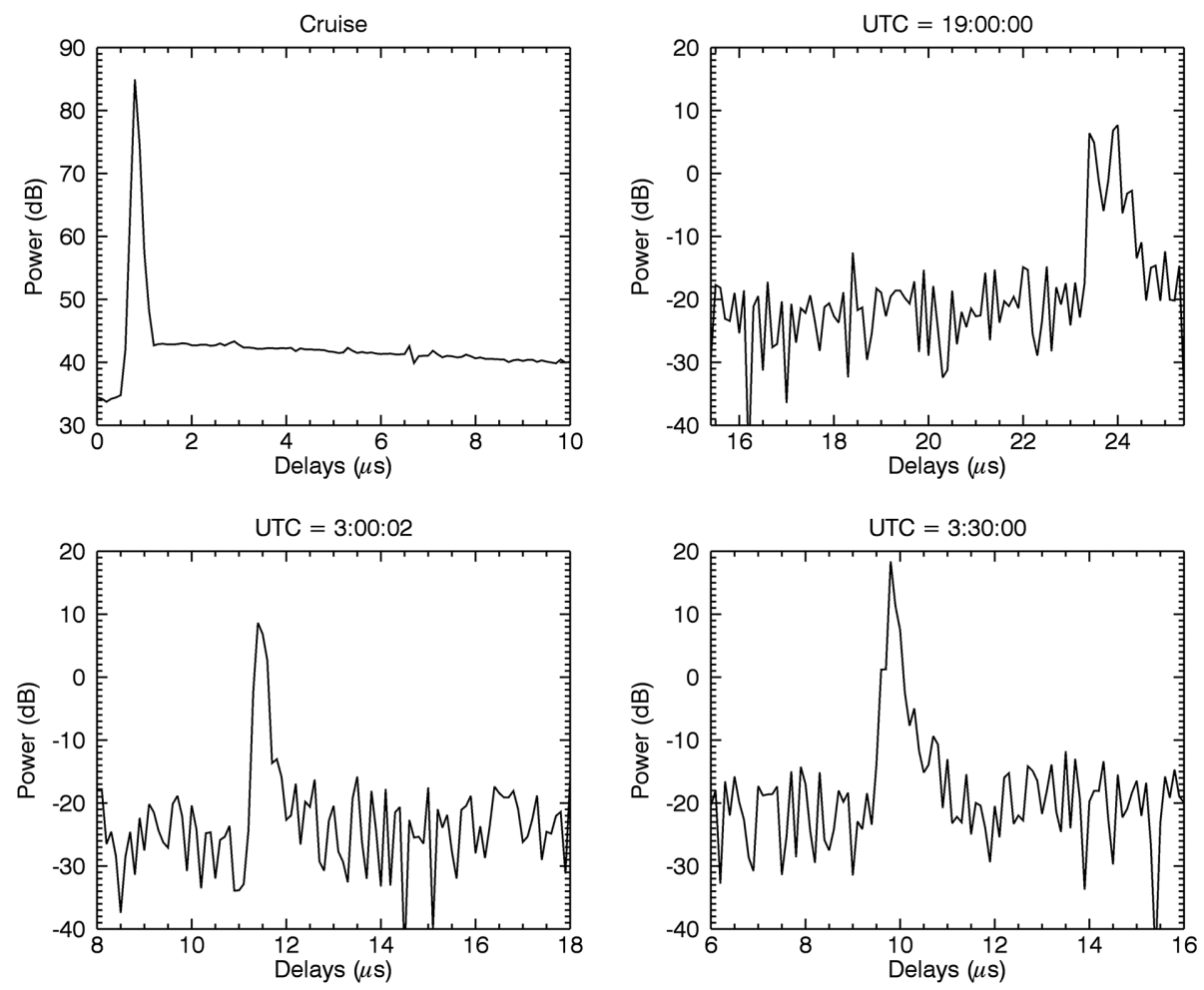

B
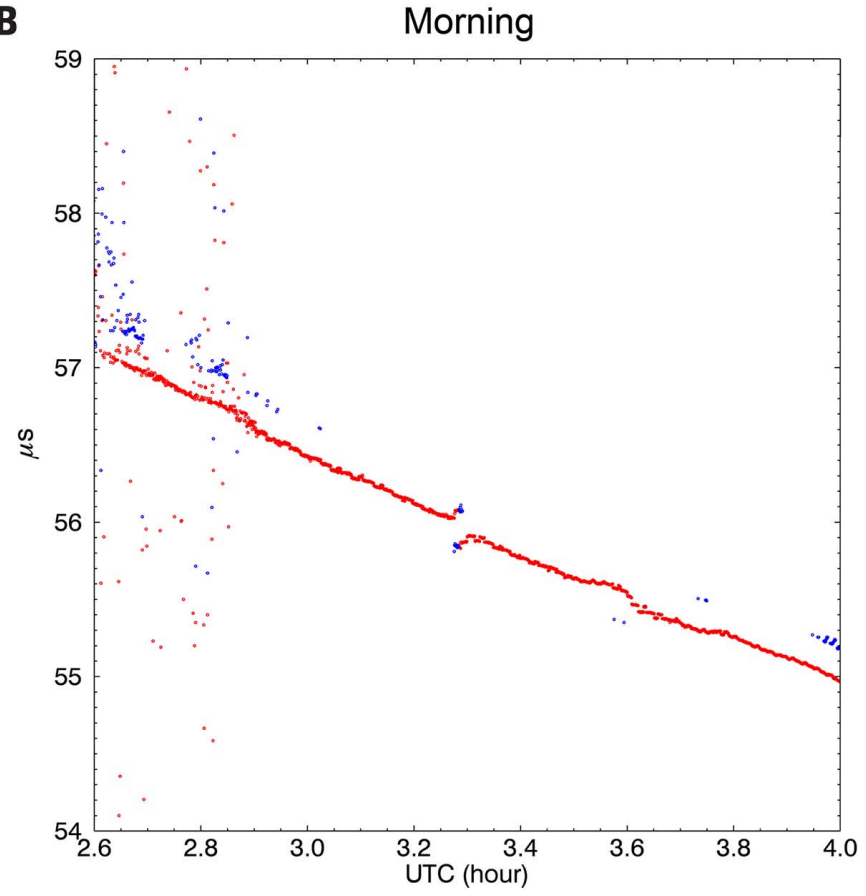

Fig. 4. Measured propagation time between Philae and Rosetta as a function of observation time. Evening (A) and morning (B) measurements. Red corresponds to the strongest signal, blue to the second strongest, and green to the third strongest. Second and third are in the interval of $6 \mathrm{~dB}$ below the red one. The dispersed dots correspond to delays not correctly detected due to the noise. 
Fig. 5. Expected landing site(s) within the strip defined by CONSERT. On the shape model of the upper lobe region corresponding to the final landing region, the hypothetical landing sites are marked by dots. Possible landing sites (low RMS of the arriving time difference between observations and simulations) are marked in yellow, with the best fit in red. Unlikely landing sites [RMS too large and impossible location for two periods (SM)] are marked in white.

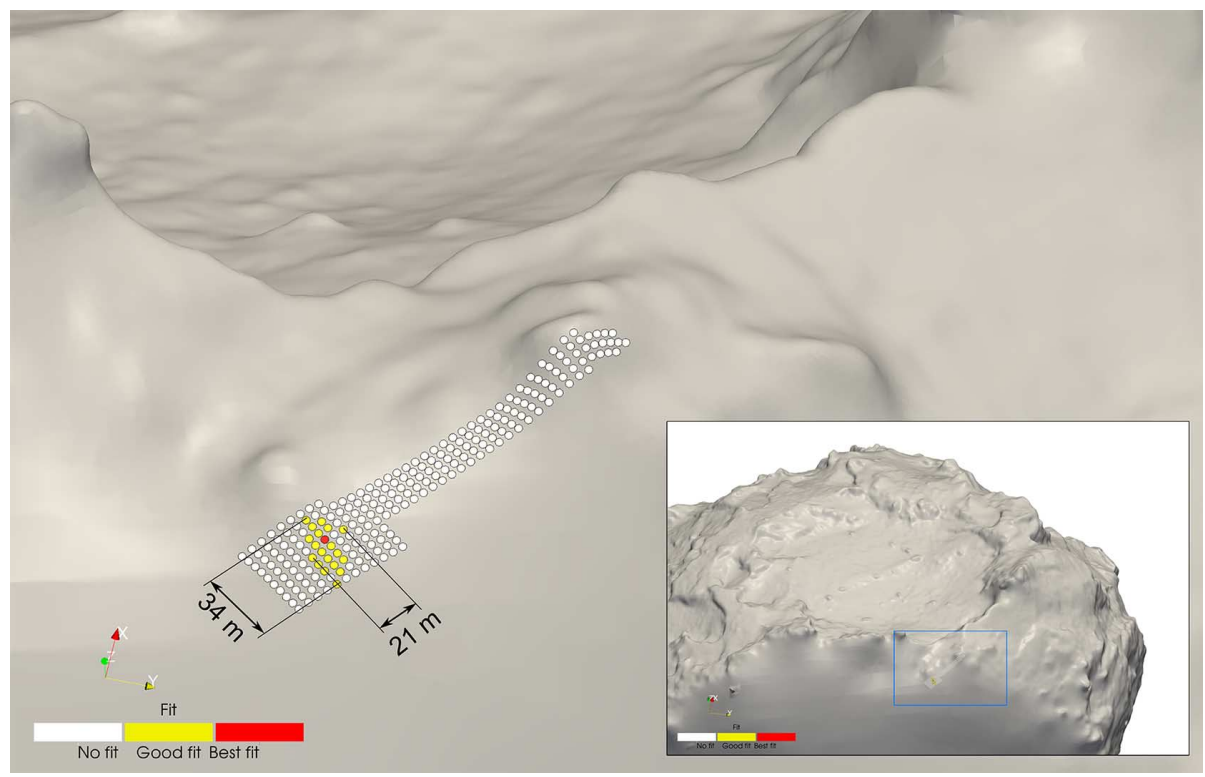

Fig. 6. Ternary diagram dust/ice/porosity volumetric fractions for cometary material. The three axes correspond to the fraction by volume of dust, ice, and vacuum (28). The vacuum volume fraction is so that the total porosity is equal to the sum of micro- and macroporosities, whereas refractory dust material is assumed to have no porosity. Constraints imposed by estimates of the comet density and dust/ice ratio coming from other instruments or observations are also indi-
A

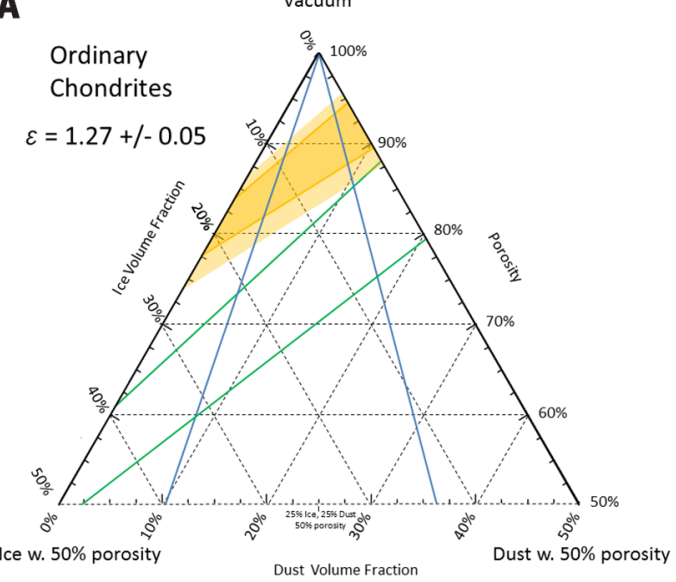

B

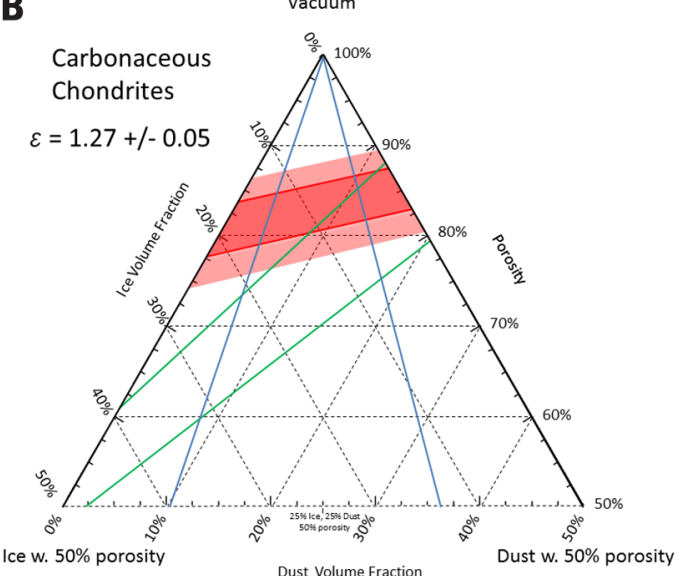

cated. The continuous lines delimit regions of $(\mathbf{A})$ and (B) (yellow and red, respectively), where the calculated permittivity is equal to 1.27 , as derived from the CONSERT observations, using a dust permittivity of ordinary chondrites (yellow region) and carbonaceus chondrites (red region). The lines (delimitating each region with darker color) correspond to limits obtained by Hashin-Shtrikman bounds. The region with lighter color indicates the influence of the error in the measurement on the permittivity of \pm 0.05 . The green lines delimit regions for the possible density, and the blue lines delimit regions for the possible dust/ice ratio (SM).

consistent with a bulk solar system composition for primitive material (24-26). They appear to be primarily composed of ferromagnesian silicates, Fe-Ni sulfides, and Fe-Ni metal. Abundant amorphous silicates were also detected in addition to the crystalline ones, consistent with mixing between processed solar system matter and interstellar matter. The accreted material could include Al-rich and Si-rich chondrule fragments together with some CAI (i.e., similar to calciumaluminium-rich-inclusions)-like fragments. These materials, combined with fine-grained components in the tracks, are analogous to components in unequilibrated chondritic meteorites and cluster interplanetary dust particles, as collected in the stratosphere of the Earth (27). Therefore, potential analog meteoritic materials for comparison with cometary dust include the ordinary and the carbonaceous chondrite groups.
To calculate the effective permittivity for the nuclei, we consider the measured permittivity on two types of chondritic meteorites. The first type consists of two ordinary chondrites (OC), and the second one consists of two carbonaceous chondrites (CC). The laboratory-measured permittivity of the samples is then used to calculate the dust/ice volume ratio using the Hashin-Shtrikman bounds for the maximum and minimum of the effective permittivity (13). Hence, we assume that in the head of comet $67 \mathrm{P}$, the mixture consists of the two most common materials in comets' chondritic dust (with permittivity of 2.6 to 2.9 for $\mathrm{CC}$ and of 4.7 to 5.6 for $\mathrm{OC}$ ) and porous ice (with permittivity of 2.8 to 3.1) [table S3 (SM)].

The permittivity derived from CONSERT data provides additional constraints to those arising from the density and ice/dust ratio, as derived from other data. Altogether, they are used to build ternary diagrams (Fig. 6 and figs. S7 to S9). Our value of the permittivity (about 1.27) excludes, as expected for primitive small bodies, the presence of ordinary chondrites in the refractory component. From laboratory measurements on material with a porosity of $30 \%$, the permittivity has to be lower than 2.9. This corresponds, using the Maxwell Garnett (13) formula for inversion, to a typical permittivity lower than 4 for a material without any porosity.

The range of dust/ice volume ratio is about 0.4 to 2.6 , and the porosity range is 75 to $85 \%$. These values correspond to the head of the comet. Deeper analysis of CONSERT based on more precise location information may reveal some variability of permittivity between different parts of the cometary head. However, it is unlikely that the values will change much from the low and narrow range of permittivity deduced here. 


\section{REFERENCES AND NOTES}

1. G. Picardi et al., Radar soundings of the subsurface of Mars. Science 310, 1925-1928 (2005). doi: 10.1126/science.1122165; pmid: 16319122

2. J. J. Plaut et al., Subsurface radar sounding of the south polar layered deposits of Mars. Science 316, 92-95 (2007). doi: 10.1126/science.1139672; pmid: 17363628

3. R. J. Phillips et al., Massive $\mathrm{CO}_{2}$ ice deposits sequestered in the south polar layered deposits of Mars. Science 332, 838-841 (2011). doi: 10.1126/science.1203091; pmid: 21512003

4. T. Ono et al., Instrumentation and observation target of the Lunar Radar Sounder (LRS) experiment on-board the SELENE spacecraft. Earth Planet. Sci. 60, 321-332 (2008) doi: 10.1186/BF03352797

5. W. Kofman et al., Comet Nucleus Sounding Experiment by Radiowave Transmission. Adv. Space Res. 21, 1589-1598 (1998). doi: 10.1016/S0273-1177(97)00952-6

6. J. Biele et al., The landing(s) of Philae and inferences on comet surface mechanical properties. Science 349, aaa9816 (2015).

7. J. Boisson et al., Radar sounding of temperate permafrost in Alaska: Analogy to the Martian midlatitude to high-latitude ice-rich terrains. J. Geophys. Res. 116, E11003 (2011). doi: 10.1029/2010JE003768

8. H. Sierks et al., On the nucleus structure and activity of comet 67P/Churyumov-Gerasimenko. Science 347, aaa1044 (2015). doi: 10.1126/science.aaa1044; pmid: 25613897

9. M. C. Festou, H. U. Keller, H. A. Weaver, Eds., Comets II (University of Arizona Press, Tucson, AZ, 2004).

10. E. Hadamcik, A. K. Sen, A. C. Levasseur-Regourd, R. Gupta, J. Lasue, Polarimetric observations of comet 67P/ChuryumovGerasimenko during its 2008-2009 apparition. Astron. Astrophys. 517, A86 (2010). doi: 10.1051/0004-6361/ 201014167

11. F. Capaccioni et al., The organic-rich surface of comet 67P/Churyumov-Gerasimenko as seen by VIRTIS/Rosetta. Science 347, aaa0628 (2015). doi: 10.1126/science.aaa0628; pmid: 25613895

12. R. Schulz et al., Comet 67P/Churyumov-Gerasimenko sheds dust coat accumulated over the past four years. Nature $\mathbf{5 1 8}$ 216-218 (2015) doi: 10.1038/nature14159; pmid: 25624103

13. A. Sihvola, Electromagnetic Mixing Formulas and Applications (The Institution of Electrical Engineers, London, 2004).

14. M. Born, E. Wolf, Principles of Optics (Pergamon Press, New York, 1970)

15. M. Benna, J.-P. Barriot, W. Kofman, Y. Barbin, Generation of 3-D synthetic data for the modeling of the CONSERT experiment (the radiotomography of comet 7P/Churyumov
Gerasimenko). IEEE Trans. Antenn. Propag. 52, 709-716 (2004). doi: 10.1109/TAP.2004.825564

16. A. Herique, W. Kofman, T. Hagfors, G. Caudal, J.-P. Ayanides, A characterization of a comet nucleus interior: Inversion of simulated radio frequency data. Planet. Space Sci. 47, 885-904 (1999). doi: 10.1016/S0032-0633(98)00144-5

17. S. R. Gough, A low temperature dielectric cell and the permittivity of hexagonal ice to $2 \mathrm{~K}$. Can. J. Chem. 50 3046-3051 (1972). doi: 10.1139/v72-483

18. E. Heggy et al., Radar properties of comets: Parametric dielectric modeling of Comet 67P/Churyumov-Gerasimenko. Icarus 221, 925-939 (2012). doi: 10.1016/j.icarus.2012.09.023

19. J. Klinger, A.-C. Levasseur-Regourd, N. Bouziani, A. Enzian, Towards a model of cometary nuclei for engineering studies for future space missions to comets. Planet. Space Sci. 44, 637-653 (1996). doi: 10.1016/0032 0633(96)00067-0

20. 0. Andersson, Dielectric relaxation of the amorphous ices J. Phys. Condens. Matter 20, 244115

(2008). doi: 10.1088/0953-8984/20/24/244115

21. E. Pettinelli et al., Frequency and time domain permittivity measurements on solid $\mathrm{CO}_{2}$ and solid $\mathrm{CO}_{2}$-soil mixtures as Martian soil simulants. J. Geophys. Res. 108 (E4), 8029 (2003). doi: 10.1029/2002JE001869

22. P. Ehrenfreund, S. B. Charnley, Organic molecules in the interstellar medium, comets, and meteorites: A voyage from dark clouds to the early Earth. Annu. Rev. Astron. Astrophys. 38, 427-483 (2000). doi: 10.1146/annurev. astro.38.1.427

23. M. S. Hanner, M. S., J.P. Bradley, in Comets II, M. C. Festou, H. U. Keller, H. A. Weaver, Eds. (University of Arizona Press, Tucson, AZ, 2004), pp. 555-564.

24. G. J. Flynn et al., Elemental compositions of comet 81P/Wild 2 samples collected by Stardust. Science 314, 1731-1735 (2006). pmid: 17170294

25. T. Stephan, Assessing the elemental composition of comet 81P/Wild 2 by analyzing dust collected by Stardust. Space Sci. Rev. 138, 247-258 (2008). doi: 10.1007/s11214 007-9291-2

26. D. Brownlee, D. Joswiak, G. Matrajt, Overview of the rocky component of Wild 2 comet samples: Insight into the early solar system, relationship with meteoritic materials and the differences between comets and asteroids. Meteorit. Planet. Sci. 47, 453-470 (2012). doi: 10.1111/j.19455100.2012.01339.x

27. D. J. Joswiak et al., Comprehensive examination of large mineral and rock fragments in Stardust tracks: Mineralogy, analogous extraterrestrial materials, and source regions.
Meteorit. Planet. Sci. 47, 471-524 (2012). doi: 10.1111/j.19455100.2012.01337.x

28. P. Kamoun, P. L. Lamy, I. Toth, A. Herique, Constraints on the subsurface structure and density of the nucleus of Comet 67P/Churyumov-Gerasimenko from Arecibo radar observations. Astron. Astrophys. 568, A21 (2014). doi: 10.1051/ 0004-6361/201423544

\section{ACKNOWLEDGMENTS}

Rosetta is an European Space Agency (ESA) mission with contributions from its member states and National

Aeronautics and Space Administration (NASA). Rosetta's Philae lander is provided by a consortium led by Deutsches Zentrum für Luft- und Raumfahrt (DLR), Max Planck Institut fur

Sonnesystemforschung (MPS), Centre National d'Etudes Spatiales (CNES), and Agenzia Spaziale Italiana (ASI). The CONSERT instrument was designed, built, and operated by IPAG, LATMOS, and MPS and was financially supported by CNES, CNRS, Université Joseph Fourier (UJF), DLR, and MPS. The authors thank the teams of Rosetta [Rosetta Mission Operation Center (RMOC) and Rosetta Science Ground Segment (RSGS)] and Philae (Rosetta Lander Control Center (LCC) and Rosetta Lander Science Operation \& Navigation Center (SONC)) for making possible the CONSERT operations. Most of the computations presented in this paper were performed using the Calcul Intensif, Modélisation, Expérimentation Numérique (CIMENT) infrastructure (https://ciment.ujf-grenoble.fr), including the Froggy platform, which is supported by the Rhône-Alpes region (GRANT CPER07_13 CIRA), the OSUG@2020 labex (reference ANR10 LABX56), the Equip@Meso project (reference ANR-10-EQPX-29-01) of the program Investissements d'Avenir supervised by the Agence Nationale pour la Recherche and France-Grille (www.france-grilles.fr). Part of this research was carried out at the Jet Propulsion Laboratory. California Institute of Technology, under a contract with the National Aeronautics and Space Administration. All CONSERT data are released through the PSA archive of ESA (www.rssd.esa.int/index.php?project=PSA\&page=rosetta)

\section{SUPPLEMENTARY MATERIALS}

www.sciencemag.org/content/349/6247/aab0639/suppl/DC1

Materials and Methods

Figs. S1 to S9

Tables S1 to S5

References (29-42)

5 March 2015; accepted 30 June 2015

10.1126/science.aab0639 


\section{Science}

\section{Properties of the 67P/Churyumov-Gerasimenko interior revealed by CONSERT radar}

Wlodek Kofman, Alain Herique, Yves Barbin, Jean-Pierre Barriot, Valérie Ciarletti, Stephen Clifford, Peter Edenhofer, Charles Elachi, Christelle Eyraud, Jean-Pierre Goutail, Essam Heggy, Laurent Jorda, Jérémie Lasue, Anny-Chantal

Levasseur-Regourd, Erling Nielsen, Pierre Pasquero, Frank Preusker, Pascal Puget, Dirk Plettemeier, Yves Rogez, Holger

Sierks, Christoph Statz, Hakan Svedhem, Iwan Williams, Sonia Zine and Jakob Van Zyl

Science 349 (6247), aab0639.

DOI: $10.1126 /$ science.aab0639

ARTICLE TOOLS

http://science.sciencemag.org/content/349/6247/aab0639

SUPPLEMENTARY

MATERIALS

http://science.sciencemag.org/content/suppl/2015/07/29/349.6247.aab0639.DC1

RELATED
CONTENT

http://science.sciencemag.org/content/sci/349/6247/493.full

file:/content

REFERENCES

This article cites 37 articles, 9 of which you can access for free http://science.sciencemag.org/content/349/6247/aab0639\#BIBL

PERMISSIONS

http://www.sciencemag.org/help/reprints-and-permissions

Use of this article is subject to the Terms of Service

Science (print ISSN 0036-8075; online ISSN 1095-9203) is published by the American Association for the Advancement of Science, 1200 New York Avenue NW, Washington, DC 20005. The title Science is a registered trademark of AAAS.

Copyright (C) 2015, American Association for the Advancement of Science 